\title{
On the Development of Refined Plate Theory for Static Bending Behavior of Functionally Graded Plates
}

\author{
Van Thom Do $\mathbb{D}^{1},{ }^{1}$ Van Vinh Pham, ${ }^{1}$ and Hoang Nam Nguyen $\mathbb{D}^{2}$ \\ ${ }^{1}$ Department of Mechanics, Le Quy Don Technical University, Hanoi City 100000, Vietnam \\ ${ }^{2}$ Modeling Evolutionary Algorithms Simulation and Artificial Intelligence, Faculty of Electrical and Electronics Engineering, \\ Ton Duc Thang University, Ho Chi Minh City 700000, Vietnam \\ Correspondence should be addressed to Hoang Nam Nguyen; nguyenhoangnam@tdtu.edu.vn
}

Received 16 January 2020; Accepted 22 February 2020; Published 7 April 2020

Academic Editor: Francesco Tornabene

Copyright (c) 2020 Van Thom Do et al. This is an open access article distributed under the Creative Commons Attribution License, which permits unrestricted use, distribution, and reproduction in any medium, provided the original work is properly cited.

This work gives information about the development of refined plate theory to study the static bending behavior of functionally graded material (FGM) plates. The significant advantage of our proposed theory is that only one unknown variable exists in its displacement formula and governing equation. To illustrate the accuracy and effectiveness of this theory, an analytical approach based on the Navier solution is employed to obtain the solution for static bending of simply supported FGM plates. A good agreement for static bending of FGM plates with other literature results has been instituted. This work also investigates the deflection, in-plane normal, and shear stresses of sinusoidally loaded FGM rectangular plates with four simply supported edges. The influence of some parameters on the bending performance of FGM plates is also carefully considered.

\section{Introduction}

Because of many advantageous features of functionally graded material (FGM), it has had numerous applications in some fields of engineering, for example, transportation, mechanics, and other structural applications, and the use of this structure is growing very rapidly, so it has attracted a great amount of concern from many researchers. Numerous theories on the plate have been established to investigate the dynamic and static responses as well as buckling of plates made of advanced composite material.

Firstly, the classical plate theory (CPT) has been applied to investigate the thin FGM plate by many researchers. In this plate theory, the transverse shear stress is neglected, so it cannot be applied to analyze moderate and thick plates. Javaheri and Eslami [1] used CPT to research the buckling of FGM plates subjected to in-plane compressive load. Mohammadi and his coworkers [2] analyzed the buckling behavior of FGM plates using CPT and the Levy solution. Thermal buckling of FGM plates was investigated by Ghannadpour et al. [3], in which, the finite strip method based on CPT was used. Damanpack and his colleagues [4] used the boundary element method based on CPT to investigate static bending of thin FGM plates. Nevertheless, CPT theory accommodates to analyze thin plates only because it does not consider the effects of shear deformation.

Secondly, to overcome the disadvantage of CPT, the Reissner-Mindlin plate theory or the first shear deformation theory (FSDT), in which the transverse shear deformation is assumed to be constant through the thickness of the plate, was developed to be capable of analyzing moderate and thick plates. Croce and Venini [5] used the Reissner-Mindlin plate theory to develop a hierarchic family of finite elements to analyze the static bending of FGM plates in a thermal environment subjected to mechanical loadings. Nguyen and his coworkers [6] applied the FSDT and the Galerkin solution to investigate the postbuckling behavior of FGM plates under a combination of mechanical loadings and thermal loadings, in which, the material properties of the plates depend on the temperature. Hosseini-Hashemi and his coworkers employed FSDT [7] and the Reissner-Mindlin plate theory [8] to investigate FGM plates in case of free vibration. Nguyen and his colleagues [9] applied the FSDT to develop a plate model to study the FGM plates. Shimpi and 
his collaborators [10] utilized a new theory based on FSDT, and Thai and his coworkers [11] established a simple theory based on FSDT which can be used to analyze the free vibration and static bending of shear deformation FGM plates. In [12], an application of a simple theory based on FSDT for the investigation of FG sandwich plates was carried out. Nguyen et al. [13] developed a refined simple FSDT to analyze the FGM plate. In this work, Nguyen presented a new shape function to describe the distribution of shear strain as well as shear stress of the plate through its thickness. A combination of the FSDT and isogeometric analysis was developed by $\mathrm{Yu}$ and Yin et al. $[14,15]$ to analyze the FGM plates. Tan-Van et al. [16] employed a modern meshless method based on FSDT to research the FGM plates. Because of its constant shear stress, FSDT needs a correction coefficient. However, this coefficient depends on the material and boundary condition.

To deal with the shortcoming of the FSDT, many scientists focus on developing higher-order shear deformation plate theories (HSDT). The first good example is the HSDT which was developed by Reddy [17] which was applied to study the plates made of FGM. The success of this theory is that it needs no correction coefficient and also the shear stresses are equal to zeros on the upper surface and the lower surface. Some other noteworthy HSDT has been carried out by many researchers such as Javaheri and Eslami [18], Bodaghi and Saidi [19], Ferreira et al. [20], and Talha and Singh [21] which have been employed to analyze FGM plates. Besides, HSDT has been combined with isogeometric for analysis of FGM plates by Tran et al. [22], composite sandwich plates by Nguyen-Xuan [23], and FGM plates by Thai et al. [24]. Zenkour [25, 26] developed generalized shear deformation theory and a HSDT with trigonometric function as well as $3 \mathrm{D}$ elasticity solutions to investigate thick FGM plates. Bui and his associates [27] applied HSDT and the finite element method (FEM) to investigate mechanical behaviors of the plate made of FGM in the high-temperature environment. Do et al. [28] used HSDT to analyze the bidirection FGM plate by FEM. Mantari and Guedes Soares [29-32] presented some other plate theories such as generalized HSDT and generalized hybrid HSDT to exponentially study FGM plates and FGM shells. Arefi and his coworkers [33] applied a two-variable sinusoidal shear deformation theory and a physical neutral surface to investigate FGM plates with the piezoelectric layer resting on the Winkler-Pasternak foundation. Tornabene and his colleagues [34] studied double-curved, singly curved, revolution shells and plates using a generalized HSDT in combination with the local generalized differential quadrature method. Mechab and his coworkers [35] applied a new theory to inspect the static bending and dynamic response of FGM plates, in which, the number of variable unknowns of the theory is four. Benachour et al. [36] investigated the free vibration of plates made of FGM by applying a new plate theory which contains four variable unknowns. Using a novel method where the transverse displacement was separated into two parts, Shimpi [37] developed a new two-variable and single-variable refined plate theory (RPT) to study isotropic plates. His idea was developed and applied to investigate orthotropic plates by Shimpi and Patel [38], Thai and Kim [39], and Mechab et al. [40] with two unknown variables. Another extended work by Shimpi et al. was presented in [41], in which a single-variable refined theory with an inertia associated term in its displacement field is applied to study free vibrations of isotropic plates. Filippi et al. [42] developed a number of plate elements based on an improved theory based on a zig-zag power function to analyze metallic and composite layered structures with viscoelastic layers. In another extended work by Carrera and his colleagues [43], a comprehensive of classical, higher-order, zig-zag, and variable kinematic shell elements were established for analysis of composite multilayered structures. Alaimo et al. [44] presented a development of an advanced analytical formulation for damped free vibration and frequency response analysis of composite plate structures embedding viscoelastic layers model. In this work, the governing equations were derived by using the principle of virtual displacement and layer-wise models which associated to linear up to fourth-order variations of the unknown variables in the thickness direction.

Because the RPT is very simple in its formulas, it has had a large number of appliances in a lot of works to investigate many kinds of the plate including isotropic and orthotropic plates. However, according to the experiences of the authors, there are no works using this theory to investigate the plate with a continuous varying of properties such as FGM. In the current work, a development is operated to modify RPT, so this theory consists of only one unknown variable, and then it is employed to investigate the static bending problem of FGM plates. The Navier solution is occupied to solve the governing equation of fully simple supported FGM plates. The proposed theory is verified by validity studies. Besides, the investigation about the effects of some parameters on the static bending behavior of the plate is also considered and debated.

\section{Material Properties of FGM Plates}

The FGM plate was made by mixing two or more different ingredients with a continuous variation in the thickness of the plate. In this study, FGM plates with the power-law function (P-FGM) and exponential function (E-FGM) as shown in Figure 1 were considered.

For a P-FGM plate, the volume of ceramic is obtained using the following formula:

$$
V_{c}=\left(\frac{1}{2}+\frac{z}{h}\right)^{p}
$$

in which $p$ is the power-law index and $h$ is the thickness of the plate. The material properties of a P-FGM can be determined as

$$
P(z)=P_{m}+\left(P_{c}-P_{m}\right) V_{c},
$$

where $P_{c}$ and $P_{m}$ are, respectively, Young's modulus or density of the ceramic and metal.

The material properties of E-FGM can be determined as

$$
P(z)=P_{0} e^{p(z / h+1 / 2)},
$$




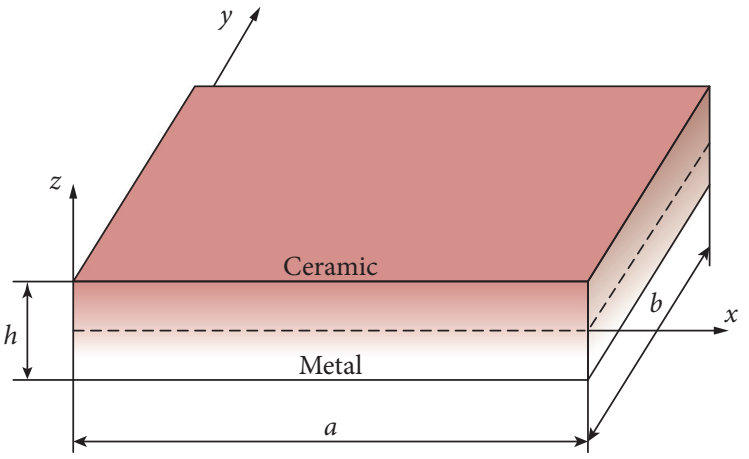

Figure 1: Model of an FGM plate.

where $P_{0}$ is Young's modulus or density of the material at the lower surface of the FGM plate and $p$ is the material parameter.

\section{New Single-Variable Refined Plate Theory}

3.1. Assumption of New Single-Variable Refined Plate Theory. The assumption of new single-variable refined plate theory based on the RPT of Shimpi [37] is given as follows:

(1) The displacement $w$ is separated into two parts, the first part is the bending part $w_{b}$ and the second part is the shear part $w_{s}$

$$
w=w_{b}+w_{s}
$$

(2) The normal stress $\sigma_{z}$ is very small in contrast with other normal stresses. Thus, the normal stress $\sigma_{z}$ is negligible. Therefore, by applying Hooke's law for a linearly elastic material, the relation between normal stresses $\sigma_{x}, \sigma_{y}$ and strains $\varepsilon_{x}, \varepsilon_{y}$ is written as

$$
\begin{gathered}
\sigma_{x}=\frac{E(z)}{1-\nu(z)^{2}}\left(\varepsilon_{x}+\nu(z) \varepsilon_{y}\right), \\
\sigma_{y}=\frac{E(z)}{1-\nu(z)^{2}}\left(\varepsilon_{y}+\nu(z) \varepsilon_{x}\right) .
\end{gathered}
$$

(3) The displacements $u$ in the $x$-direction and $v$ in the $y$-direction are divided into two parts, which are the bending part and the shear part:

$$
\begin{aligned}
& u=u_{b}+u_{s}, \\
& v=v_{b}+v_{s} .
\end{aligned}
$$

The first parts $u_{b}$ as well as $v_{b}$ are similar to the displacements given by the CPT, which are

$$
\begin{aligned}
& u_{b}=-z \frac{\partial w_{b}}{\partial x} \\
& v_{b}=-z \frac{\partial w_{b}}{\partial y} .
\end{aligned}
$$

The second parts $u_{s}$ and $v_{s}$ give rise to shear strain $\gamma_{x z}, \gamma_{y z}$ and therefore to shear stresses $\tau_{x z}, \tau_{y z}$ which have distribution through the depth and are equal to zero at the top and bottom surfaces of the plate. Consequently, the expression for $u_{s}$ and $v_{s}$ can be obtained as

$$
\begin{aligned}
& u_{s}=f(z) \frac{\partial w_{s}}{\partial x}, \\
& v_{s}=f(z) \frac{\partial w_{s}}{\partial y},
\end{aligned}
$$

where $f(z)$ is the shear distributed profile function, and this function describes the spreading of the shear stresses $\tau_{x z}, \tau_{y z}$ throughout the thickness. In this study, the shear distributed shape function $f(z)$ of Shimpi [37] as given in the following formula is used:

$$
f(z)=-\frac{5 z^{3}}{3 h^{2}}+\frac{z}{4}
$$

It is noticed that $u_{s}$ and $v_{s}$ do not provide to moment $M_{x}, M_{y}$, and $M_{x y}$.

3.2. Expressions for Displacement of Proposed Theory. By using the assumptions which are discussed above, for the case of the plate, the expressions of displacement are

$$
\begin{aligned}
& u=-z \frac{\partial w_{b}}{\partial x}+f(z) \frac{\partial w_{s}}{\partial x} \\
& v=-z \frac{\partial w_{b}}{\partial y}+f(z) \frac{\partial w_{s}}{\partial y}, \\
& w=w_{b}+w_{s} .
\end{aligned}
$$

The formulas for strain fields are

$$
\begin{aligned}
\varepsilon_{x} & =-z \frac{\partial^{2} w_{b}}{\partial x^{2}}+f(z) \frac{\partial^{2} w_{s}}{\partial x^{2}}, \\
\varepsilon_{y} & =-z \frac{\partial^{2} w_{b}}{\partial y^{2}}+f(z) \frac{\partial^{2} w_{s}}{\partial y^{2}}, \\
\gamma_{x y} & =-z\left[2 \frac{\partial^{2} w_{b}}{\partial x \partial y}\right]+f(z)\left[2 \frac{\partial^{2} w_{s}}{\partial x \partial y}\right], \\
\gamma_{x z} & =\frac{\partial w_{s}}{\partial x} g(z), \\
\gamma_{y z} & =\frac{\partial w_{s}}{\partial y} g(z),
\end{aligned}
$$

where $g(z)=1+f^{\prime}(z)$.

The formulas for normal stresses $\sigma_{x}$ and $\sigma_{y}$ are obtained by using equation (11) and equation (5). The formulas for $\tau_{x y}, \tau_{x z}$, and $\tau_{y z}$ are calculated by using equation (11) and the following constitutive equations:

$$
\begin{aligned}
& \tau_{x y}=G(z) \gamma_{x y}, \\
& \tau_{x z}=G(z) \gamma_{x z}, \\
& \tau_{y z}=G(z) \gamma_{y z} .
\end{aligned}
$$


Subsequently, the expressions for normal stresses and shear stresses are

$$
\begin{aligned}
\sigma_{x} & =\frac{E(z)}{1-v(z)^{2}}\left[-z \frac{\partial^{2} w_{b}}{\partial x^{2}}+f(z) \frac{\partial^{2} w_{s}}{\partial x^{2}}+v(z)\left(-z \frac{\partial^{2} w_{b}}{\partial y^{2}}+f(z) \frac{\partial^{2} w_{s}}{\partial y^{2}}\right)\right], \\
\sigma_{y} & =\frac{E(z)}{1-v(z)^{2}}\left[-z \frac{\partial^{2} w_{b}}{\partial y^{2}}+f(z) \frac{\partial^{2} w_{s}}{\partial y^{2}}+v(z)\left(-z \frac{\partial^{2} w_{b}}{\partial x^{2}}+f(z) \frac{\partial^{2} w_{s}}{\partial x^{2}}\right)\right], \\
\tau_{x y} & =\frac{E(z)}{2(1+v(z))}\left(-2 z \frac{\partial^{2} w_{b}}{\partial x \partial y}+2 f(z) \frac{\partial^{2} w_{s}}{\partial x \partial y}\right) . \\
\tau_{y z} & =\frac{g(z) E(z)}{2(1+v(z))} \frac{\partial w_{s}}{\partial y} \\
\tau_{x z} & =\frac{g(z) E(z)}{2(1+v(z))} \frac{\partial w_{s}}{\partial x} .
\end{aligned}
$$

Equation (13) and equation (14) can be rewritten as

$$
\begin{aligned}
\sigma_{x}= & \frac{-z E(z)}{1-\nu(z)^{2}}\left(\frac{\partial^{2} w_{b}}{\partial x^{2}}+v(z) \frac{\partial^{2} w_{b}}{\partial y^{2}}\right)+\frac{f(z) E(z)}{1-v^{2}} \\
& \cdot\left(\frac{\partial^{2} w_{s}}{\partial x^{2}}+v(z) \frac{\partial^{2} w_{s}}{\partial y^{2}}\right), \\
\sigma_{y}= & \frac{-z E(z)}{1-\nu(z)^{2}}\left(\frac{\partial^{2} w_{b}}{\partial y^{2}}+v(z) \frac{\partial^{2} w_{b}}{\partial x^{2}}\right)+\frac{f(z) E(z)}{1-v^{2}} \\
& \cdot\left(\frac{\partial^{2} w_{s}}{\partial y^{2}}+v(z) \frac{\partial^{2} w_{s}}{\partial x^{2}}\right), \\
\tau_{x y}= & \frac{-z E(z)}{1-\nu(z)^{2}}(1-v(z)) \frac{\partial^{2} w_{b}}{\partial x \partial y}+\frac{f(z) E(z)}{1-v(z)^{2}} \\
& \cdot(1-v(z)) \frac{\partial^{2} w_{s}}{\partial x \partial y} . \\
\tau_{y z}= & \frac{g(z) E(z)}{2(1+v(z))} \frac{\partial w_{s}}{\partial y}, \\
\tau_{x z}= & \frac{g(z) E(z)}{2(1+v(z))} \frac{\partial w_{s}}{\partial x} .
\end{aligned}
$$

The moments and shear forces are obtained as

$$
\begin{aligned}
\left\{\begin{array}{c}
M_{x} \\
M_{y} \\
M_{x y}
\end{array}\right\}=\int_{-h / 2}^{h / 2} z\left\{\begin{array}{c}
\sigma_{x} \\
\sigma_{y} \\
\tau_{x y}
\end{array}\right\} \mathrm{d} z, \\
\left\{\begin{array}{l}
Q_{x} \\
Q_{y}
\end{array}\right\}=\int_{-h / 2}^{h / 2}\left\{\begin{array}{c}
\tau_{x z} \\
\tau_{y z}
\end{array}\right\} \mathrm{d} z .
\end{aligned}
$$

Based on the assumption of the present theory, the moments and shear forces are

$$
\begin{aligned}
M_{x} & =\int_{-h / 2}^{h / 2} \frac{-z^{2} E(z)}{1-v(z)^{2}}\left[\frac{\partial^{2} w_{b}}{\partial x^{2}}+v(z) \frac{\partial^{2} w_{b}}{\partial y^{2}}\right] \mathrm{d} z, \\
M_{y} & =\int_{-h / 2}^{h / 2} \frac{-z^{2} E(z)}{1-\nu(z)^{2}}\left[\frac{\partial^{2} w_{b}}{\partial y^{2}}+v(z) \frac{\partial^{2} w_{b}}{\partial x^{2}}\right] \mathrm{d} z, \\
M_{x y} & =\int_{-h / 2}^{h / 2} \frac{-z^{2} E(z)}{\left(1-v(z)^{2}\right)}\left[(1-v(z)) \frac{\partial^{2} w_{b}}{\partial x \partial y}\right] \mathrm{d} z . \\
Q_{x} & =\int_{-h / 2}^{h / 2} \frac{E(z)}{2(1+v(z))} g(z) \frac{\partial w_{s}}{\partial x} \mathrm{~d} z, \\
Q_{y} & =\int_{-h / 2}^{h / 2} \frac{E(z)}{2(1+v(z))} g(z) \frac{\partial w_{s}}{\partial y} \mathrm{~d} z .
\end{aligned}
$$

After integrating equation (19) and equation (20) over the thickness $h$, the moments and shear forces are expressed as the following formulas:

$$
\begin{aligned}
& M_{x}=-\alpha \frac{\partial^{2} w_{b}}{\partial x^{2}}-\alpha_{1} \frac{\partial^{2} w_{b}}{\partial y^{2}}, \\
& M_{y}=-\alpha \frac{\partial^{2} w_{b}}{\partial y^{2}}-\alpha_{1} \frac{\partial^{2} w_{b}}{\partial x^{2}}, \\
& M_{x y}=-\alpha \frac{\partial^{2} w_{b}}{\partial x \partial y}+\alpha_{1} \frac{\partial^{2} w_{b}}{\partial x \partial y} . \\
& Q_{x}=\beta \frac{\partial w_{s}}{\partial x}, \\
& Q_{y}=\beta \frac{\partial w_{s}}{\partial y} .
\end{aligned}
$$

The coefficients $\alpha, \alpha_{1}$, and $\beta$ are calculated as 


$$
\begin{gathered}
\alpha=\int_{-h / 2}^{h / 2} \frac{z^{2} E(z)}{1-v(z)^{2}} \mathrm{~d} z, \\
\alpha_{1}=\int_{-h / 2}^{h / 2} \frac{z^{2} v(z) E(z)}{1-v(z)^{2}} \mathrm{~d} z, \\
\beta=\int_{-h / 2}^{h / 2} \frac{E(z)}{2(1+v(z))} g(z) \mathrm{d} z .
\end{gathered}
$$

We can see that if $\nu$ does not depend on $z$-direction, then $\alpha_{1}=v \alpha$. The expressions for moments do not include $w_{s}$, and the expressions for shear forces do not include $w_{b}$.

The equilibrium equations are

$$
\begin{aligned}
& \frac{\partial \sigma_{x}}{\partial x}+\frac{\partial \tau_{x y}}{\partial y}+\frac{\partial \tau_{x z}}{\partial z}=0 \\
& \frac{\partial \sigma_{y}}{\partial y}+\frac{\partial \tau_{x y}}{\partial x}+\frac{\partial \tau_{y z}}{\partial z}=0 \\
& \frac{\partial \tau_{x z}}{\partial x}+\frac{\partial \tau_{y z}}{\partial y}+\frac{\partial \sigma_{z}}{\partial z}=0 .
\end{aligned}
$$

Multiply the first two equations with respect to $z$ and then integrating with respect to $z$ through the thickness, we get

$$
\begin{gathered}
\frac{\partial M_{x}}{\partial x}+\frac{\partial M_{x y}}{\partial y}=Q_{x}, \\
\frac{\partial M_{x y}}{\partial x}+\frac{\partial M_{y}}{\partial y}=Q_{y}, \\
\frac{\partial Q_{x}}{\partial x}+\frac{\partial Q_{y}}{\partial y}=-q .
\end{gathered}
$$

Substituting equation (21) and equation (22) into equation (27), one gets

$$
\begin{array}{r}
-\frac{\partial}{\partial x}\left[\alpha \frac{\partial^{2} w_{b}}{\partial x^{2}}+\alpha_{1} \frac{\partial^{2} w_{b}}{\partial y^{2}}\right]-\frac{\partial}{\partial y}\left[\left(\alpha-\alpha_{1}\right) \frac{\partial^{2} w_{b}}{\partial x \partial y}\right]-\beta \frac{\partial w_{s}}{\partial x}=0, \\
-\frac{\partial}{\partial x}\left[\left(\alpha-\alpha_{1}\right) \frac{\partial^{2} w_{b}}{\partial x \partial y}\right]-\frac{\partial}{\partial y}\left[\alpha \frac{\partial^{2} w_{b}}{\partial y^{2}}+\alpha_{1} \frac{\partial^{2} w_{b}}{\partial x^{2}}\right]-\beta \frac{\partial w_{s}}{\partial y}=0, \\
\beta \frac{\partial^{2} w_{s}}{\partial x^{2}}+\beta \frac{\partial^{2} w_{s}}{\partial y^{2}}+q=0,
\end{array}
$$

and the first two equations of equation (28) become

$$
\begin{aligned}
& \frac{\partial w_{s}}{\partial x}=-\frac{\alpha}{\beta} \frac{\partial}{\partial x}\left(\frac{\partial^{2} w_{b}}{\partial x^{2}}+\frac{\partial^{2} w_{b}}{\partial y^{2}}\right), \\
& \frac{\partial w_{s}}{\partial y}=-\frac{\alpha}{\beta} \frac{\partial}{\partial y}\left(\frac{\partial^{2} w_{b}}{\partial x^{2}}+\frac{\partial^{2} w_{b}}{\partial y^{2}}\right),
\end{aligned}
$$

or

$$
w_{s}=-\frac{\alpha}{\beta}\left(\frac{\partial^{2} w_{b}}{\partial x^{2}}+\frac{\partial^{2} w_{b}}{\partial y^{2}}\right)=\chi\left(\nabla w_{b}\right),
$$

in which $\chi=-\alpha / \beta$ and $\nabla$ is the Laplace operator, $\nabla=\partial^{2} / \partial x^{2}+\partial^{2} / \partial y^{2}$

It is clear that equation (30) reveals the relation between the bending part and the shear part of transverse displacement, and it is similar to Shimpi's theory. However, in this theory, the coefficient $\chi$ not only depends on the thickness but also depends on the varying material properties, and it is an implicit integral expression. This is a significant particular different point of this theory in comparison with Shimpi's theory. Consequently, this theory is compatible to investigate FGM plates. If Young's modulus and Poisson ratio of the material are constant, the implicit integral expression $\chi$ becomes an explicit expression of Young's modulus, Poisson ratio, and the thickness $h$, so it is completely identical with the plate theory of Shimpi.

By means of introducing equation (30) into equation (10), the new displacement formulas of the proposed theory are

$$
\begin{aligned}
& u=-z \frac{\partial w_{b}}{\partial x}+f(z) \frac{\partial}{\partial x}\left[\chi\left(\nabla w_{b}\right)\right] \\
& v=-z \frac{\partial w_{b}}{\partial y}+f(z) \frac{\partial}{\partial y}\left[\chi\left(\nabla w_{b}\right)\right] \\
& w=w_{b}+\chi\left(\nabla w_{b}\right) .
\end{aligned}
$$

It can see that the formulas of displacement of the proposed theory consist of only one unknown variable, the bending component $w_{b}$.

3.3. Expressions for Strains, Stresses, Moments, and Shear Forces. By the way of introducing equation (31) into equation (11), new expressions for strains of proposed theory are obtained as

$$
\begin{aligned}
\varepsilon_{x} & =-z \frac{\partial^{2} w_{b}}{\partial x^{2}}+f(z) \frac{\partial^{2}}{\partial x^{2}}\left[\chi\left(\nabla w_{b}\right)\right], \\
\varepsilon_{y} & =-z \frac{\partial^{2} w_{b}}{\partial y^{2}}+f(z) \frac{\partial^{2}}{\partial y^{2}}\left[\chi\left(\nabla w_{b}\right)\right], \\
\gamma_{x y} & =-z\left[2 \frac{\partial^{2} w_{b}}{\partial x \partial y}\right]+f(z)\left\{2 \frac{\partial^{2}}{\partial x \partial y}\left[\chi\left(\nabla w_{b}\right)\right]\right\}, \\
\gamma_{y z} & =g(z) \frac{\partial}{\partial y}\left[\chi\left(\nabla w_{b}\right)\right], \\
\gamma_{x z} & =g(z) \frac{\partial}{\partial x}\left[\chi\left(\nabla w_{b}\right)\right] .
\end{aligned}
$$

By introducing equation (31) into equation (15) and equation (16), the expressions for stresses of the proposed theory can be obtained as 


$$
\begin{aligned}
\sigma_{x}= & \frac{-z E(z)}{1-\nu(z)^{2}}\left(\frac{\partial^{2} w_{b}}{\partial x^{2}}+v(z) \frac{\partial^{2} w_{b}}{\partial y^{2}}\right)+\frac{f(z) E(z)}{1-v^{2}} \\
& \cdot\left\{\frac{\partial^{2}}{\partial x^{2}}\left[\chi\left(\nabla w_{b}\right)\right]+v(z) \frac{\partial^{2}}{\partial y^{2}}\left[\chi\left(\nabla w_{b}\right)\right]\right\}, \\
\sigma_{y}= & \frac{-z E(z)}{1-v(z)^{2}}\left(\frac{\partial^{2} w_{b}}{\partial y^{2}}+v(z) \frac{\partial^{2} w_{b}}{\partial x^{2}}\right)+\frac{f(z) E(z)}{1-v^{2}} \\
& \cdot\left\{\frac{\partial^{2}}{\partial y^{2}}\left[\chi\left(\nabla w_{b}\right)\right]+v(z) \frac{\partial^{2}}{\partial x^{2}}\left[\chi\left(\nabla w_{b}\right)\right]\right\}, \\
\tau_{x y}= & \frac{-z E(z)}{1+v(z)} \frac{\partial^{2} w_{b}}{\partial x \partial y}+\frac{f(z) E(z)}{1+v(z)} \frac{\partial^{2}}{\partial x \partial y}\left[\chi\left(\nabla w_{b}\right)\right] . \\
\tau_{y z}= & \frac{g(z) E(z)}{2(1+v(z))} \frac{\partial}{\partial y}\left[\chi\left(\nabla w_{b}\right)\right], \\
\tau_{x z}= & \frac{g(z) E(z)}{2(1+v(z))} \frac{\partial}{\partial x}\left[\chi\left(\nabla w_{b}\right)\right] .
\end{aligned}
$$

By introducing equation (31) into equation (21) and equation (22), the expressions for moments as well as shear forces are taken as

$$
\begin{aligned}
M_{x} & =-\alpha \frac{\partial^{2} w_{b}}{\partial x^{2}}-\alpha \frac{\partial^{2} w_{b}}{\partial y^{2}}, \\
M_{y} & =-\alpha \frac{\partial^{2} w_{b}}{\partial y^{2}}-\alpha_{1} \frac{\partial^{2} w_{b}}{\partial x^{2}}, \\
M_{x y} & =-\alpha \frac{\partial^{2} w_{b}}{\partial x \partial y}+\alpha_{1} \frac{\partial^{2} w_{b}}{\partial x \partial y} . \\
Q_{x} & =\beta \frac{\partial}{\partial x}\left[\chi\left(\nabla w_{b}\right)\right], \\
Q_{y} & =\beta \frac{\partial}{\partial y}\left[\chi\left(\nabla w_{b}\right)\right] .
\end{aligned}
$$

3.4. Governing Equation. Substituting equation (35) and equation (36) into equation (27), it becomes

$$
\alpha\left(\nabla \nabla w_{b}\right)-q=0 .
$$

This is a simple governing differential equation of the static bending problem of a plate. It is clear that the governing equation of the plate consists of only one unknown variable which is the bending component $w_{b}$. The coefficient $\alpha$ is an implicit integral expression of Young's modulus, Poisson ratio, and the thickness $h$. In case of the homogeneous plate, coefficient $\alpha$ becomes an explicit expression of Young's modulus, Poisson ratio, and the thickness $h$, and the governing differential equation of this theory is completely identical to that of Shimpi.

\section{Analytical Solutions}

In this work, the Navier procedure is applied to analyze a rectangular FGM plate, and the boundary condition of the plate is simply supported at all edges. The solution is implicit as the following formula:

$$
w_{b}(x, y)=\sum_{k=1}^{\infty} \sum_{r=1}^{\infty} W_{b k r} \sin \varphi x \sin \psi y,
$$

where $\varphi=k \pi / a, \psi=r \pi / b$, and $W_{b k r}$ are the quantities which must be determined. The distributed load can expand in the form of double trigonometric series as

$$
q(x, y)=\sum_{k=1}^{\infty} \sum_{r=1}^{\infty} Q_{k r} \sin \varphi x \sin \psi y,
$$

in which

$$
Q_{k r}=\frac{4}{a b} \int_{0}^{a} \int_{0}^{b} q(x, y) \sin \psi x \sin \psi y \mathrm{~d} x \mathrm{~d} y .
$$

Substituting equation (38) and equation (39) into equation (37), the analytical solution for the bending component of transverse displacement is

$$
W_{b k r}=\frac{Q_{k r}}{\alpha\left(\varphi^{2}+\psi^{2}\right)^{2}}
$$

and with sinusoidally distributed load, the coefficient $Q_{k r}$ is

$$
Q_{\mathrm{kr}}=q_{0}, \quad k=r=1,
$$

and with uniformly distributed load, the coefficient $Q_{k r}$ is

$$
Q_{k r}=\frac{16 q_{0}}{k r \pi^{2}}
$$

\section{Illustrative Examples and Discussion}

Continuously, some examples will be considered to demonstrate the accurateness and effectiveness of the proposed theory which will be applied to investigate some problems of FGM plates. The following nondimensional formulas are used in the rest of this work:

$$
\begin{gathered}
\bar{w}=\frac{10 E_{c} h^{3}}{q_{0} a^{4}} w\left(\frac{a}{2}, \frac{b}{2}\right), \\
\bar{\sigma}_{x}=\frac{h}{q_{0} a} \sigma_{x}\left(\frac{a}{2}, \frac{b}{2}, \frac{h}{2}\right), \\
\bar{\sigma}_{y}=\frac{h}{q_{0} a} \sigma_{y}\left(\frac{a}{2}, \frac{b}{2}, \frac{h}{3}\right), \\
\bar{\tau}_{x y}=\frac{h}{q_{0} a} \tau_{x y}\left(0,0,-\frac{h}{3}\right), \\
\bar{\tau}_{x z}=\frac{h}{q_{0} a} \tau_{x z}\left(0, \frac{b}{2}, 0\right), \\
\bar{\tau}_{y z}=\frac{h}{q_{0} a} \tau_{x z}\left(\frac{a}{2}, 0, \frac{h}{6}\right) .
\end{gathered}
$$


TABLE 1: Effects of power-law index on the nondimensional stresses and displacements of a P-FGM square plate $(a / h=10)$ subjected to a uniformly distributed load.

\begin{tabular}{|c|c|c|c|c|c|c|c|}
\hline$p$ & Source & $\bar{w}$ & $\bar{\sigma}_{x}$ & $\bar{\sigma}_{y}$ & $\bar{\tau}_{y z}$ & $\bar{\tau}_{x z}$ & $\bar{\tau}_{x y}$ \\
\hline \multirow{2}{*}{ Ceramic } & [26] & 0.4665 & 2.8932 & 1.9103 & 0.4429 & 0.5114 & 1.2850 \\
\hline & Present & 0.4666 & 2.8917 & 1.9107 & 0.4497 & 0.5059 & 1.2771 \\
\hline \multirow{2}{*}{1} & [26] & 0.9287 & 4.4745 & 2.1692 & 0.5446 & 0.5114 & 1.1143 \\
\hline & Present & 0.9260 & 4.4403 & 2.1655 & 0.5124 & 0.4688 & 1.1417 \\
\hline \multirow{2}{*}{2} & [26] & 1.1940 & 5.2296 & 2.0338 & 0.5734 & 0.4700 & 0.9907 \\
\hline & Present & 1.1875 & 5.1781 & 2.0320 & 0.5021 & 0.4010 & 1.0290 \\
\hline \multirow{2}{*}{3} & {$[26]$} & 1.3200 & 5.6108 & 1.8593 & 0.5629 & 0.4367 & 1.0047 \\
\hline & Present & 1.3098 & 5.5473 & 1.8593 & 0.4726 & 0.3573 & 1.0518 \\
\hline \multirow{2}{*}{4} & [26] & 1.3890 & 5.8915 & 1.7197 & 0.5346 & 0.4204 & 1.0298 \\
\hline & Present & 1.3762 & 5.8199 & 1.7210 & 0.4414 & 0.3382 & 1.0827 \\
\hline \multirow{2}{*}{5} & [26] & 1.4356 & 6.1504 & 1.6104 & 0.5031 & 0.4177 & 1.0451 \\
\hline & Present & 1.4215 & 6.0739 & 1.6124 & 0.4153 & 0.3359 & 1.1008 \\
\hline \multirow{2}{*}{6} & [26] & 1.4727 & 6.4043 & 1.5214 & 0.4755 & 0.4227 & 1.0536 \\
\hline & Present & 1.4583 & 6.3254 & 1.5238 & 0.3963 & 0.3432 & 1.1098 \\
\hline \multirow{2}{*}{7} & [26] & 1.5049 & 6.6547 & 1.4467 & 0.4543 & 0.4310 & 1.0589 \\
\hline & Present & 1.4911 & 6.5751 & 1.4492 & 0.3841 & 0.3550 & 1.1142 \\
\hline \multirow[b]{2}{*}{8} & [26] & 1.5343 & 6.8999 & 1.3829 & 0.4392 & 0.4399 & 1.0628 \\
\hline & Present & 1.5215 & 6.8209 & 1.3854 & 0.3774 & 0.3683 & 1.1165 \\
\hline \multirow{2}{*}{9} & [26] & 1.5617 & 7.1383 & 1.3283 & 0.4291 & 0.4481 & 1.0662 \\
\hline & Present & 1.5501 & 7.0607 & 1.3308 & 0.3749 & 0.3814 & 1.1178 \\
\hline \multirow{2}{*}{10} & [26] & 1.5876 & 7.3689 & 1.2820 & 0.4227 & 0.4552 & 1.0694 \\
\hline & Present & 1.5774 & 7.2931 & 1.2843 & 0.3752 & 0.3936 & 1.1187 \\
\hline \multirow[b]{2}{*}{ Metal } & [26] & 2.5327 & 2.8932 & 1.9103 & 0.4429 & 0.5114 & 1.2850 \\
\hline & Present & 2.5329 & 2.8917 & 1.9107 & 0.4497 & 0.5059 & 1.2771 \\
\hline
\end{tabular}

TABLE 2: Effects of power-law index on the nondimensional stresses and displacements of a P-FGM square plate $(a / h=10)$ subjected to a sinusoidally distributed load.

\begin{tabular}{|c|c|c|c|c|c|c|c|}
\hline$p$ & Source & $\bar{w}$ & $\bar{\sigma}_{x}$ & $\bar{\sigma}_{y}$ & $\bar{\tau}_{y z}$ & $\bar{\tau}_{x z}$ & $\bar{\tau}_{x y}$ \\
\hline \multirow{2}{*}{ Ceramic } & [26] & 0.2960 & 1.9955 & 1.3121 & 0.2132 & 0.2462 & 0.7065 \\
\hline & Present & 0.2961 & 1.9943 & 1.3124 & 0.2122 & 0.2387 & 0.7067 \\
\hline \multirow{2}{*}{1} & {$[26]$} & 0.5889 & 3.0870 & 1.4894 & 0.2622 & 0.2462 & 0.6110 \\
\hline & Present & 0.5870 & 3.0533 & 1.4856 & 0.2418 & 0.2212 & 0.6148 \\
\hline \multirow{2}{*}{2} & [26] & 0.7573 & 3.6094 & 1.3954 & 0.2763 & 0.2265 & 0.5441 \\
\hline & Present & 0.7529 & 3.5585 & 1.3935 & 0.2369 & 0.1892 & 0.5493 \\
\hline \multirow{2}{*}{3} & {$[26]$} & 0.8377 & 3.8742 & 1.2748 & 0.2715 & 0.2107 & 0.5525 \\
\hline & Present & 0.8307 & 3.8114 & 1.2746 & 0.2230 & 0.1686 & 0.5589 \\
\hline \multirow{2}{*}{4} & [26] & 0.8819 & 4.0693 & 1.1783 & 0.2580 & 0.2029 & 0.5667 \\
\hline & Present & 0.8731 & 3.9984 & 1.1794 & 0.2083 & 0.1596 & 0.5740 \\
\hline \multirow{2}{*}{5} & [26] & 0.9118 & 4.2488 & 1.1029 & 0.2429 & 0.2017 & 0.5755 \\
\hline & Present & 0.9021 & 4.1731 & 1.1048 & 0.1960 & 0.1585 & 0.5831 \\
\hline \multirow[b]{2}{*}{6} & [26] & 0.9356 & 4.4244 & 1.0417 & 0.2296 & 0.2041 & 0.5803 \\
\hline & Present & 0.9257 & 4.3462 & 1.0439 & 0.1870 & 0.1620 & 0.5881 \\
\hline \multirow{2}{*}{7} & [26] & 0.9562 & 4.5971 & 0.9903 & 0.2194 & 0.2081 & 0.5834 \\
\hline & Present & 0.9468 & 4.5184 & 0.9927 & 0.1813 & 0.1675 & 0.5911 \\
\hline \multirow{2}{*}{8} & [26] & 0.9750 & 4.7661 & 0.9466 & 0.2121 & 0.2124 & 0.5856 \\
\hline & Present & 0.9662 & 4.6880 & 0.9489 & 0.1781 & 0.1738 & 0.5932 \\
\hline \multirow{2}{*}{9} & [26] & 0.9925 & 4.9303 & 0.9092 & 0.2072 & 0.2164 & 0.5875 \\
\hline & Present & 0.9846 & 4.8536 & 0.9115 & 0.1769 & 0.1800 & 0.5949 \\
\hline \multirow{2}{*}{10} & {$[26]$} & 1.0089 & 5.0890 & 0.8775 & 0.2041 & 0.2198 & 0.5894 \\
\hline & Present & 1.0020 & 5.0142 & 0.8797 & 0.1770 & 0.1858 & 0.5964 \\
\hline \multirow{2}{*}{ Metal } & [26] & 1.6070 & 1.9955 & 1.3121 & 0.2132 & 0.2462 & 0.7065 \\
\hline & Present & 1.6072 & 1.9943 & 1.3124 & 0.2122 & 0.2387 & 0.7067 \\
\hline
\end{tabular}


TABLE 3: Effects of material parameter $p$ on the nondimensional displacements of an E-FGM square plate $(a / h=10)$ subjected to a sinusoidally distributed load.

\begin{tabular}{|c|c|c|c|c|c|c|c|c|}
\hline \multirow{2}{*}{$a / h$} & \multirow{2}{*}{$b / a$} & \multirow{2}{*}{ Method } & \multicolumn{6}{|c|}{$p$} \\
\hline & & & 0.1 & 0.3 & 0.5 & 0.7 & 1 & 1.5 \\
\hline \multirow{15}{*}{2} & \multirow{4}{*}{1} & [25] & 0.5769 & 0.5247 & 0.4766 & 0.4324 & 0.3727 & 0.2890 \\
\hline & & [29] & 0.6363 & 0.5752 & 0.5195 & 0.4687 & 0.4018 & 0.3079 \\
\hline & & Present & 0.6422 & 0.5795 & 0.5214 & 0.4679 & 0.3959 & 0.2965 \\
\hline & & [25] & 1.1944 & 1.0859 & 0.9864 & 0.8952 & 0.7727 & 0.6017 \\
\hline & \multirow[t]{2}{*}{2} & [29] & 1.2776 & 1.1553 & 1.0441 & 0.9431 & 0.8093 & 0.6238 \\
\hline & & Present & 1.2835 & 1.1589 & 1.0442 & 0.9390 & 0.7980 & 0.6037 \\
\hline & \multirow{4}{*}{3} & [25] & 1.4430 & 1.3116 & 1.1913 & 1.0812 & 0.9334 & 0.7275 \\
\hline & & [29] & 1.5341 & 1.3874 & 1.2540 & 1.1329 & 0.9725 & 0.7506 \\
\hline & & Present & 1.5400 & 1.3907 & 1.2535 & 1.1278 & 0.9594 & 0.7276 \\
\hline & & {$[25]$} & 1.5515 & 1.4101 & 1.2807 & 1.1624 & 1.0035 & 0.7824 \\
\hline & \multirow[t]{3}{*}{4} & [29] & 1.6458 & 1.4885 & 1.3455 & 1.2157 & 1.0437 & 0.8060 \\
\hline & & Present & 1.6518 & 1.4917 & 1.3447 & 1.2101 & 1.0298 & 0.7818 \\
\hline & & [25] & 1.6065 & 1.4601 & 1.3261 & 1.2035 & 1.0391 & 0.8102 \\
\hline & \multirow[t]{2}{*}{5} & [29] & 1.7025 & 1.5397 & 1.3919 & 1.2576 & 1.0798 & 0.8340 \\
\hline & & Present & 1.7084 & 1.5429 & 1.3910 & 1.2518 & 1.0655 & 0.8092 \\
\hline \multirow{15}{*}{4} & \multirow{4}{*}{1} & [25] & 0.3490 & 0.3168 & 0.2875 & 0.2608 & 0.2253 & 0.1805 \\
\hline & & [29] & 0.3602 & 0.3259 & 0.2949 & 0.2668 & 0.2295 & 0.1785 \\
\hline & & Present & 0.3605 & 0.3259 & 0.2944 & 0.2657 & 0.2275 & 0.1751 \\
\hline & & {$[25]$} & 0.8153 & 0.7395 & 0.6708 & 0.6085 & 0.5257 & 0.4120 \\
\hline & \multirow[t]{3}{*}{2} & [29] & 0.8325 & 0.7534 & 0.6819 & 0.6173 & 0.5319 & 0.4150 \\
\hline & & Present & 0.8328 & 0.7532 & 0.6810 & 0.6155 & 0.5285 & 0.4094 \\
\hline & & [25] & 1.0134 & 0.9190 & 0.8335 & 0.7561 & 0.6533 & 0.5121 \\
\hline & \multirow[t]{3}{*}{3} & [29] & 1.0325 & 0.9345 & 0.8459 & 0.7659 & 0.6601 & 0.5154 \\
\hline & & Present & 1.0329 & 0.9343 & 0.8449 & 0.7638 & 0.6562 & 0.5091 \\
\hline & & [25] & 1.1012 & 0.9985 & 0.9056 & 0.8215 & 0.7098 & 0.5564 \\
\hline & \multirow[t]{3}{*}{4} & [29] & 1.1211 & 1.0147 & 0.9186 & 0.8317 & 0.7169 & 0.5599 \\
\hline & & Present & 1.1215 & 1.0145 & 0.9174 & 0.8295 & 0.7128 & 0.5532 \\
\hline & & {$[25]$} & 1.1459 & 1.0391 & 0.9424 & 0.8548 & 0.7386 & 0.5790 \\
\hline & \multirow[t]{2}{*}{5} & [29] & 1.1663 & 1.0556 & 0.9556 & 0.8653 & 0.7458 & 0.5825 \\
\hline & & Present & 1.1666 & 1.0553 & 0.9544 & 0.8629 & 0.7416 & 0.5758 \\
\hline
\end{tabular}

5.1. Validity Study. In this first example, a P-FGM plate is investigated, and the plate is made of two components: the first component is aluminum ( $\mathrm{Al})$ and the second component is alumina $\left(\mathrm{Al}_{2} \mathrm{O}_{3}\right)$. The plate has the length of $a$, the width of $b$, and length-to-thickness ratio of $a / h=10$. The load which acts upon the plate are uniformly distributed load or sinusoidally load. The material properties of aluminum are $E_{m}=70 \mathrm{GPa}$ and $v_{m}=0.3$ and those of alumina are $E_{c}=380 \mathrm{GPa}$ and $v_{c}=0.3$. The nondimensional values of transverse displacement and nondimensional values as well as distribution of stresses are compared with those of the study by Zenkour [26]. The results of Zenkour are calculated using the sinusoidal shear deformation theory (SSDT). Table 1 illustrates the comparison of the results of the plate under uniform load, and Table 2 indicates the comparison of the results in the case of the sinusoidally loaded plate. According to Tables 1 and 3, it can see that the results of the current work are very similar to the solutions of Zenkour using SSDT.

Incessantly, an E-FGM plate which has a length-tothickness ratio of $a / h=2$ and $a / h=4$ is considered. Young's modulus of it is obtained by a function of the exponent, while Poisson's ratios are assumed constant and equal to 0.3 . The plate is subjected to bisinusoidal load. Table 2 indicates the numerical results which are calculated by the proposed theory and those of Zenkour [25] using a solution of $3 \mathrm{D}$ elasticity theory as well as Mantari and Guedes Soares [29] using HSDT. According to this illustration, the numerical results of the plates using the proposed theory are similar with published data for both moderate and thick plates.

According to above two examples, it can be concluded that a new theory which is developed by authors is accurate and effective, and also it can be applied to investigate FGM plates in both cases of thin and thick plates.

5.2. Static Bending Behavior of FGM Plates. sthis section, a $\mathrm{P}-\mathrm{FGM}$ plate which is made of aluminum (Al) as the metal and zirconia $\left(\mathrm{ZrO}_{2}\right)$ as the ceramic subjected to sinusoidally distributed load is investigated. The material properties of constituents are

$$
\begin{aligned}
& \mathrm{Al}: E_{m}=70 \mathrm{GPa}, v_{m}=0.3, \\
& \mathrm{ZrO}_{2}: E_{c}=200 \mathrm{GPa}, v_{c}=0.3 .
\end{aligned}
$$

The numerical results which are computed by using the new theory are publicized in Table 4 . In this example, both thick and thin plates are considered for several values of other parameters. 
TABLE 4: Effects of power-index on the nondimensional displacements of a P-FGM rectangular plate subjected to a sinusoidally distributed load.

\begin{tabular}{|c|c|c|c|c|c|c|c|c|}
\hline \multirow{2}{*}{$a / h$} & \multirow{2}{*}{$a / b$} & \multicolumn{7}{|c|}{$p$} \\
\hline & & Ceramic & 1 & 2 & 3 & 5 & 10 & Metal \\
\hline \multirow{3}{*}{2} & 1 & 0.6754 & 1.0136 & 1.1975 & 1.3152 & 1.4641 & 1.6393 & 1.9298 \\
\hline & 0.5 & 1.3497 & 2.0538 & 2.4071 & 2.6199 & 2.8844 & 3.2084 & 3.8563 \\
\hline & 0.2 & 1.7964 & 2.7480 & 3.2110 & 3.4826 & 3.8175 & 4.2352 & 5.1325 \\
\hline \multirow{3}{*}{4} & 1 & 0.3790 & 0.5909 & 0.6830 & 0.7316 & 0.7894 & 0.8673 & 1.0830 \\
\hline & 0.5 & 0.8755 & 1.3774 & 1.5840 & 1.6861 & 1.8049 & 1.9732 & 2.5015 \\
\hline & 0.2 & 1.2264 & 1.9351 & 2.2216 & 2.3602 & 2.5200 & 2.7506 & 3.5041 \\
\hline \multirow{3}{*}{10} & 1 & 0.2961 & 0.4725 & 0.5390 & 0.5682 & 0.6004 & 0.6511 & 0.8459 \\
\hline & 0.5 & 0.7428 & 1.1880 & 1.3535 & 1.4247 & 1.5026 & 1.6273 & 2.1222 \\
\hline & 0.2 & 1.0669 & 1.7075 & 1.9446 & 2.0460 & 2.1567 & 2.3349 & 3.0482 \\
\hline \multirow{3}{*}{100} & 1 & 0.2804 & 0.4502 & 0.5118 & 0.5374 & 0.5648 & 0.6104 & 0.8012 \\
\hline & 0.5 & 0.7177 & 1.1523 & 1.3100 & 1.3754 & 1.4456 & 1.5621 & 2.0506 \\
\hline & 0.2 & 1.0368 & 1.6645 & 1.8924 & 1.9867 & 2.0882 & 2.2565 & 2.9622 \\
\hline
\end{tabular}

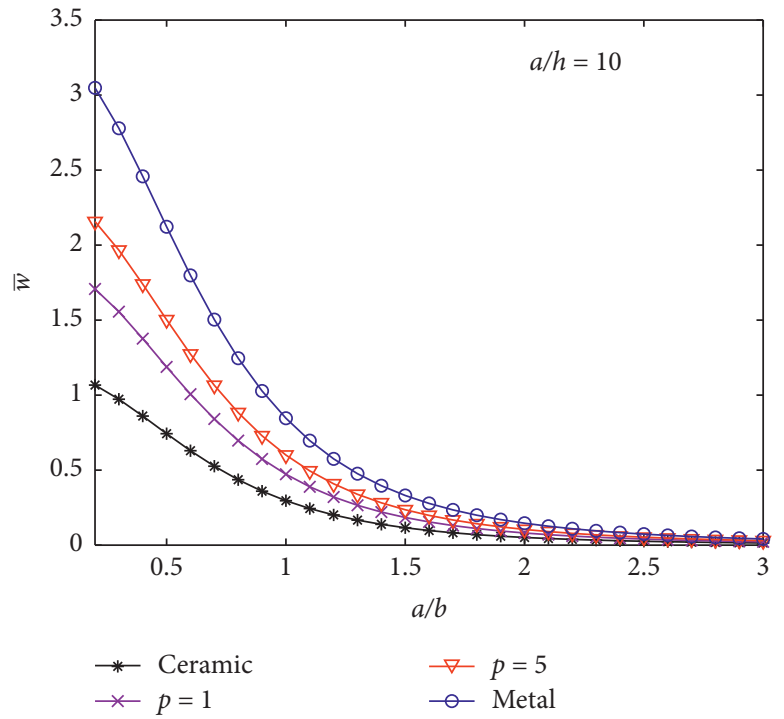

Figure 2: Nondimensional deflection $\bar{w}$ of the P-FGM plate related with $a / b$.

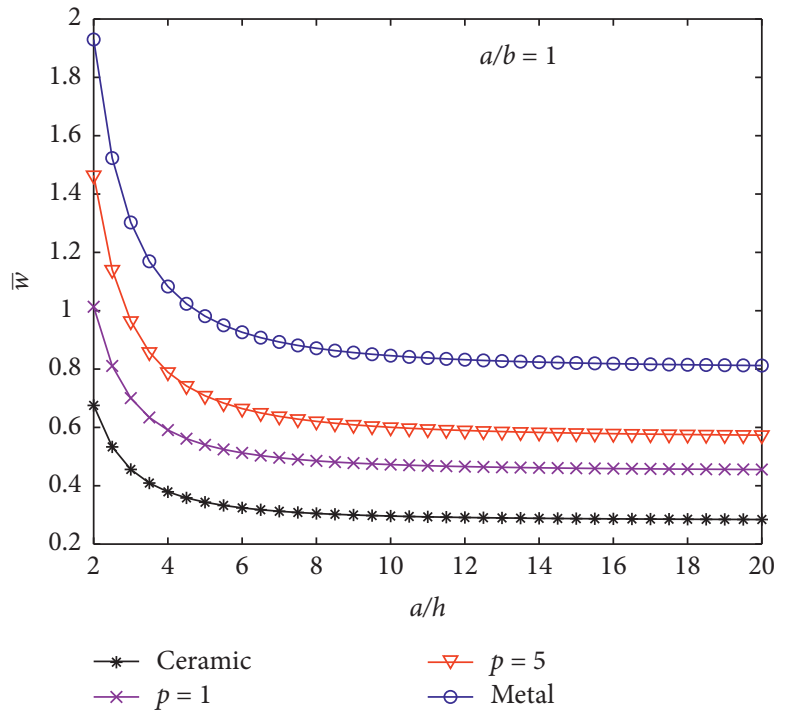

FIGURE 3: Nondimensional deflection $\bar{w}$ of the P-FGM plate related with $a / h$.

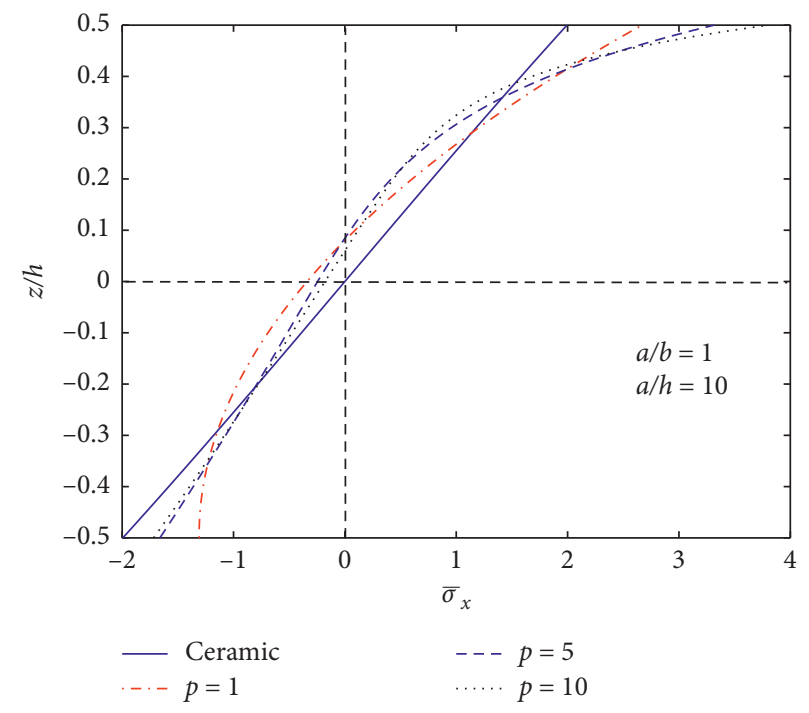

FIgure 4: Distribution of $\bar{\sigma}_{x}$ along the depth of the P-FGM plate.

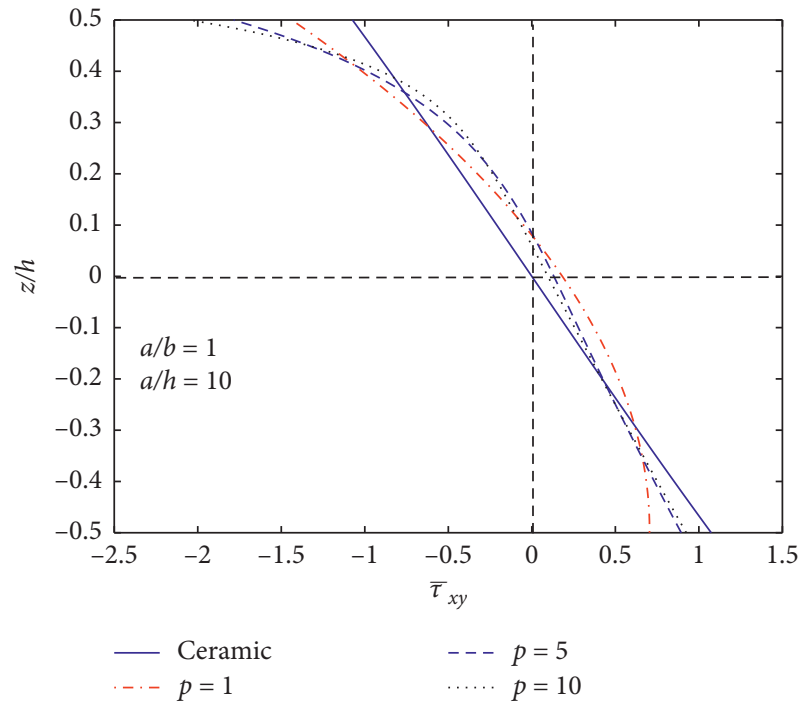

Figure 5: Distribution of $\bar{\tau}_{x y}$ along the depth of the P-FGM plate. 


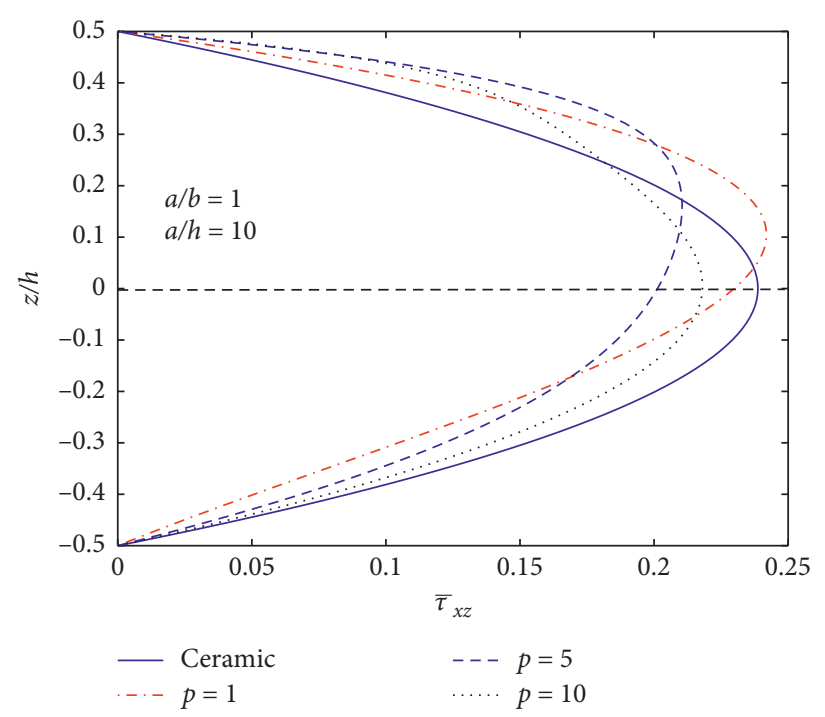

FIgURe 6: Distribution of $\bar{\tau}_{x z}$ along the depth of the P-FGM plate.

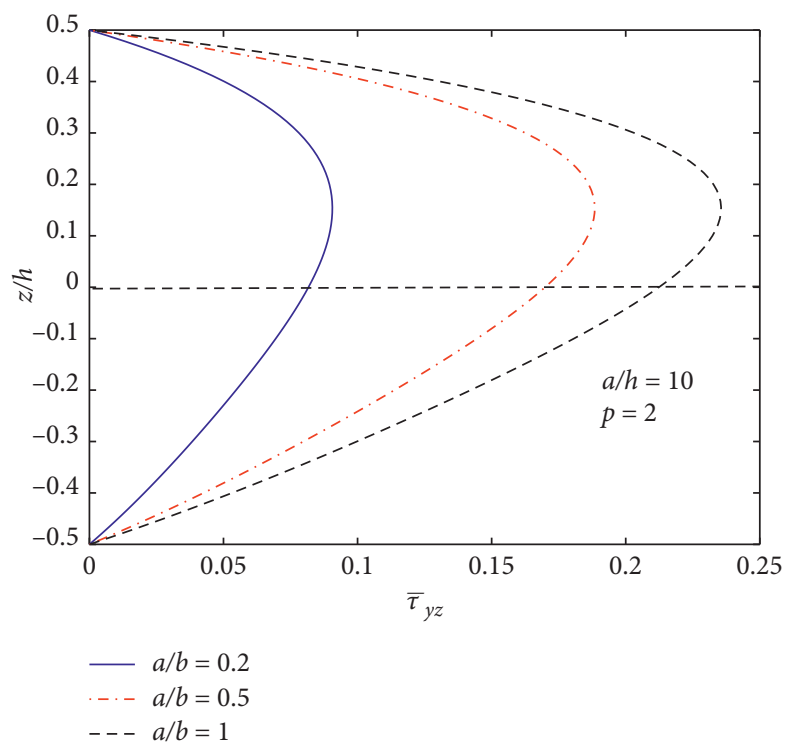

FIgURe 7: Distribution of $\bar{\tau}_{y z}$ along the depth of the P-FGM plate.

The results in Table 4 show that the deflection of the P-FGM plate rises as there is an increase the power-law index, especially when it varies in a range of 0 to 2 . This also shows that the risk ceramic plate is harder than the risk metal plate. It is true that the highest value of the deflection is achieved in the case of the metallic plate, but the smallest value of the deflection is achieved in the case of the ceramic plate. The reason is that the elastic modulus of the metal is less than that of ceramic. In addition, when the aspect ratio increases, the deflection decreases. Moreover, the deflection of the plate increases when $a / h$ is increased. Figures 2 and 3 show more clearly the effects of $a / b$ and $a / h$ on the deflection of the P-FGM plate. Figure 2 shows the nondimensional results of deflection of the P-FGM plate gathering to the ratio of $a / h$. According to Figure 2, the side-to-thickness ratio has principal responsibility to the bending behavior of the plate. As $a / h$ ratio rises in a range of 2 to 4 , the deflection decreases rapidly. When it is greater than 4 , the deflection approximate unchanged with its increase. The exhibition in Figure 3 shows that the deflection decreases when the aspect ratio increases especially when this ratio varies from 0 to 1 .

Figures 4-7 performed the distributions of the stresses in $z$-direction of the plate. As exhibited in Figures 4 and 5, the in-plane normal stresses and the longitudinal tangential stress are nonlinearly distributed along with the thickness of the P-FGM plate; the neutral surface is not placed at the midsurface of the P-FGM plate, and it differs from a homogeneous plate. On the upper surface, the 

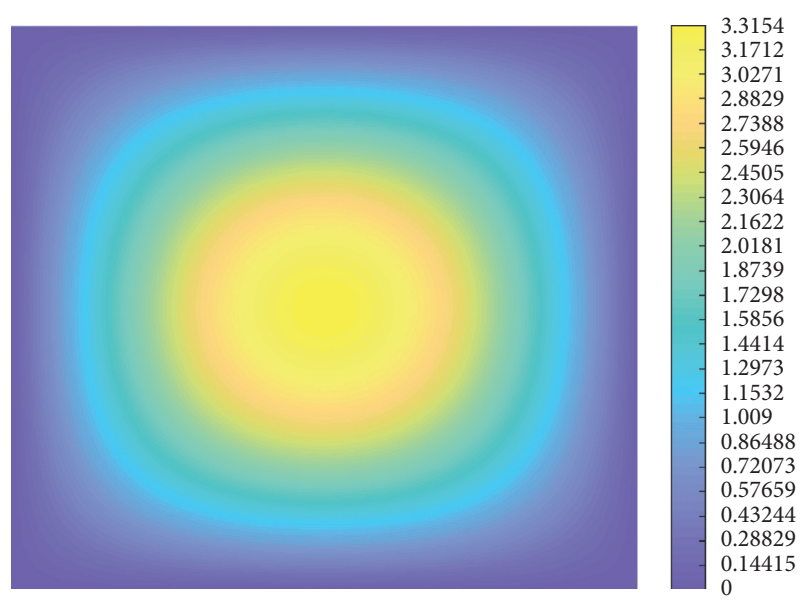

(a)

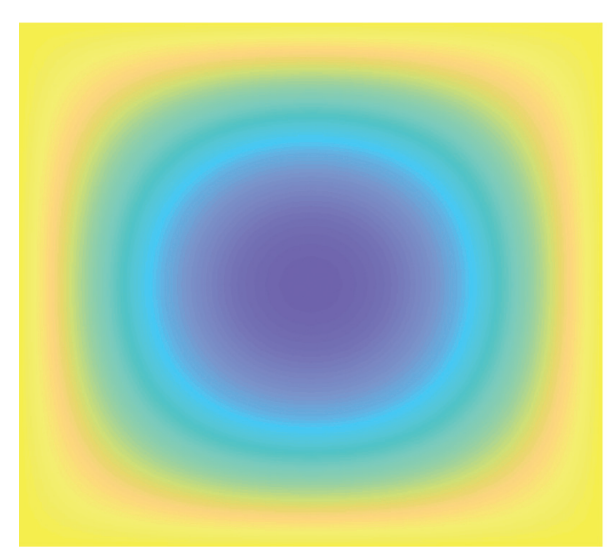

(c)

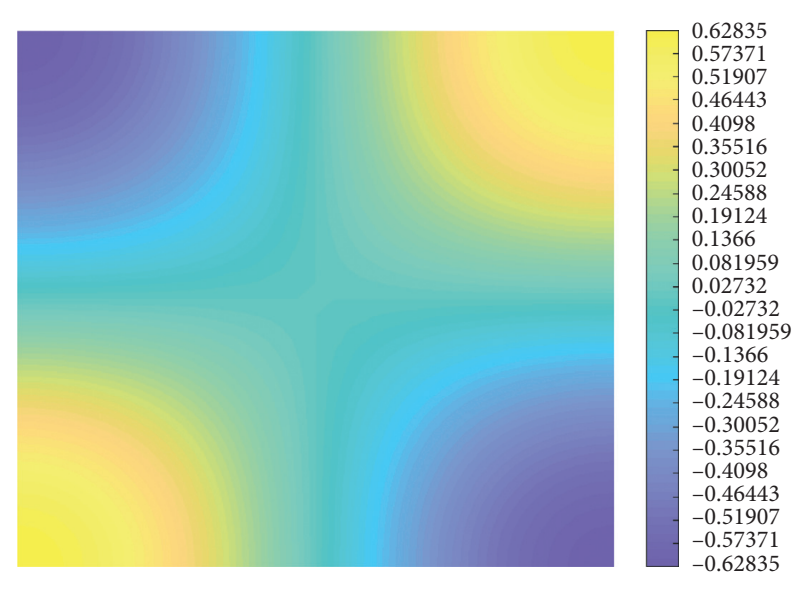

(b)

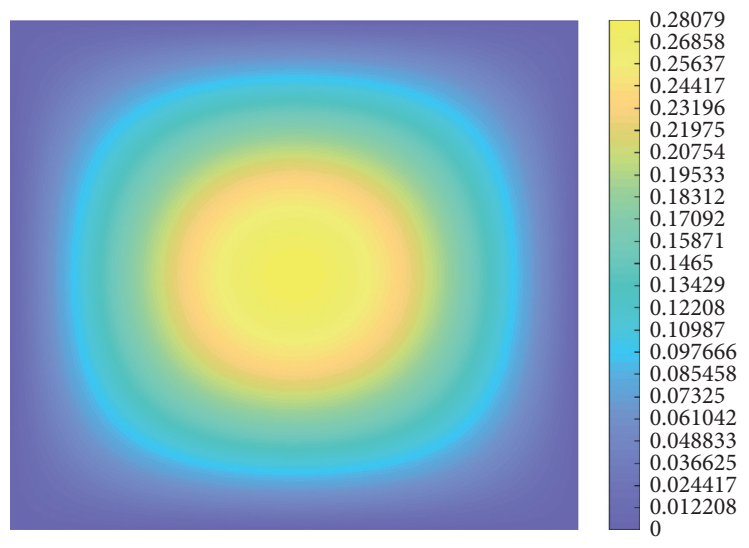

(d)

FIGURE 8: Distribution of nondimensional stresses in the $x y$ plane of the P-FGM plate with $a=b, a / h=10$, and $p=5$ : (a) in-plane normal stress $\bar{\sigma}_{x}(x, y, h / 2)$; (b) in-plane longitudinal tangential stress $\bar{\tau}_{x y}(x, y,-h / 3)$; (c) shear stress $\bar{\tau}_{x z}(x, y, 0)$; $(\mathrm{d})$ shear stress $\bar{\tau}_{y z}(x, y, h / 6)$.

normal stress is tensile, while it is compressive stress on the lower surface. In addition, the maximum value of the tensile longitudinal tangential stress occurs at a point on the lower surface, while the maximum compressive longitudinal tangential stress occurs at a point on the upper surface. Figures 6 and 7 represent the dissemination of the shear stresses throughout the depth of the plate. It can easily see that the plate is free of shear strains and stresses at both upper and lower surfaces. Nevertheless, the shear stresses of an FGM plate are not parabolic, and they are not highest on the midplane as in the case of the homogeneous plate. When the aspect ratio increases, the shear stress increases as given in Figure 7.

The nondimensional distribution of normal stress $\bar{\sigma}_{x}(x, y, h / 2)$, in-plane longitudinal tangential stress $\bar{\tau}_{x y}(x, y,-h / 3)$, shear stress $\bar{\tau}_{x z}(x, y, 0)$, and shear stress $\bar{\tau}_{y z}(x, y, h / 6)$ in the $x y$ plane of the plate are shown in Figure 8. It shows that the normal stress and shear stresses are symmetric, but the longitudinal tangential stress is unsymmetrical. The in-plane normal stress $\left(\bar{\sigma}_{x}\right)$ is maximum at the central point and is minimum at four corners of the plate, and this also appears with shear stress $\left(\bar{\tau}_{y z}\right)$. It is clear that $\bar{\sigma}_{x}$ is compressive stress over the upper surface of the plate. In opposite sides, the shear stress $\left(\bar{\tau}_{x z}\right)$ is maximum at four corners of the plate and is minimum at the central point. Not to mention, the maximum longitudinal tangential stress $\left(\bar{\tau}_{x y}\right)$ appears at two opposite corners of the plate while the minimum value of longitudinal tangential stress appears at two other corners.

\section{Conclusions}

In this study, a new single-variable refined plate theory which consists of only one unknown variable in its displacement formula and its governing equation was developed. The proposed theory was successfully verified against the available literature in many cases of P-FGM and E-FGM. The proposed theory was applied to analyze P-FGM plates subjected to mechanical load in several cases of thick and thin plates. Furthermore, a large parametric investigation was aimed at checking the sensitivity of the static bending of P-FGM plates to different mechanical and geometrical properties. This comprehensive parametric investigation was presented as benchmark results for future works. 


\section{Data Availability}

The data used to support the findings of this study are included within the article.

\section{Conflicts of Interest}

The authors declare that they have no conflicts of interest.

\section{Acknowledgments}

Hoang Nam Nguyen gratefully acknowledges the support of the Ton Duc Thang University Foundation for Science and Technology Development, under grant number 2019/TDTPLHDLV.

\section{References}

[1] R. Javaheri and M. R. Eslami, "Buckling of functionally graded plates under in-plane compressive loading," ZAMM, vol. 82, no. 4, pp. 277-283, 2002.

[2] M. Mohammadi, A. R. Saidi, and E. Jomehzadeh, "Levy solution for buckling analysis of functionally graded rectangular plates," Applied Composite Materials, vol. 17, no. 2, pp. 81-93, 2010.

[3] S. A. M. Ghannadpour, H. R. Ovesy, and M. Nassirnia, "Buckling analysis of functionally graded plates under thermal loadings using the finite strip method," Computers \& Structures, vol. 108-109, pp. 93-99, 2012.

[4] A. R. Damanpack, M. Bodaghi, H. Ghassemi, and M. Sayehbani, "Boundary element method applied to the bending analysis of thin functionally graded plates," Latin American Journal of Solids and Structures, vol. 10, no. 3, pp. 549-570, 2013.

[5] L.-D. Croce and P. Venini, "Finite elements for functionally graded Reissner-Mindlin plates," Computer Methods in Applied Mechanics and Engineering, vol. 193, no. 9, pp. 705-725, 2004.

[6] D.-D. Nguyen and V.-T. Hoang, "Mechanical and thermal post-buckling of shear-deformable FGM plates with temperature-dependent properties," Mechanics of Composite Materials, vol. 46, no. 5, pp. 461-476, 2010.

[7] S. Hosseini-Hashemi, H. Rokni Damavandi Taher, H. Akhavan, and M. Omidi, "Free vibration of functionally graded rectangular plates using first-order shear deformation plate theory," Applied Mathematical Modelling, vol. 34, no. 5, pp. 1276-1291, 2010.

[8] S. Hosseini-Hashemi, M. Fadaee, and S. R. Atashipour, "A new exact analytical approach for free vibration of ReissnerMindlin functionally graded rectangular plates," International Journal of Mechanical Sciences, vol. 53, no. 1, pp. 11-22, 2011.

[9] T.-K. Nguyen, K. Sab, and G. Bonnet, "First-order shear deformation plate models for functionally graded materials," Composite Structures, vol. 83, no. 1, pp. 25-36, 2008.

[10] R. P. Shimpi, H. G. Patel, and H. Arya, "New first-order shear deformation plate theories," Journal of Applied Mechanics, vol. 74, no. 3, pp. 523-533, 2007.

[11] H.-T. Thai and D.-H. Choi, "A simple first-order shear deformation theory for the bending and free vibration analysis of functionally graded plates," Composite Structures, vol. 101, pp. 332-340, 2013.

[12] H.-T. Thai, T.-K. Nguyen, T. P. Vo, and J. Lee, "Analysis of functionally graded sandwich plates using a new first-order shear deformation theory," European Journal of Mechanics-A/Solids, vol. 45, pp. 211-225, 2014.

[13] H.-N. Nguyen, T.-T. Hong, P.-V. Vinh, N.-D. Quang, and D.-V. Thom, "A refined simple first-order shear deformation theory for static bending and free vibration analysis of advanced composite plates," Materials, vol. 12, no. 15, pp. 1-25, 2019.

[14] T. T. Yu, S. Yin, T. Q. Bui, and S. Hirose, "A simple FSDTbased isogeometric analysis for geometrically nonlinear analysis of functionally graded plates," Finite Elements in Analysis and Design, vol. 96, pp. 1-10, 2015.

[15] S. Yin, J. S. Hale, T. Yu, T. Q. Bui, and S. P. A. Bordas, "Isogeometric locking-free plate element: a simple first order shear deformation theory for functionally graded plates," Composite Structures, vol. 118, pp. 121-138, 2014.

[16] V. Tan-Van, N. Ngoc-Hung, A. Khosravifard, M.-R. Hematiyan, S. Tanaka, and T.-Q. Bui, "A simple FSDTbased meshfree method for analysis of functionally graded plates," Engineering Analysis with Boundary Elements, vol. 79, pp. 1-12, 2017.

[17] J.-N. Reddy, "Analysis of functionally graded plates," International Journal for Numerical Methods in Engineering, vol. 47, no. 1-3, pp. 663-684, 2000.

[18] R. Javaheri and M. R. Eslami, "Thermal buckling of functionally graded plates based on higher order theory," Journal of Thermal Stresses, vol. 25, no. 7, pp. 603-625, 2002.

[19] M. Bodaghi and A. R. Saidi, "Levy-type solution for buckling analysis of thick functionally graded rectangular plates based on the higher-order shear deformation plate theory," Applied Mathematical Modelling, vol. 34, no. 11, pp. 3659-3673, 2010.

[20] A. J. M. Ferreira, R. C. Batra, C. M. C. Roque, L. F. Qian, and P. A. L. S. Martins, "Static analysis of functionally graded plates using third-order shear deformation theory and a meshless method," Composite Structures, vol. 69, no. 4, pp. 449-457, 2005.

[21] M. Talha and B. N. Singh, "Static response and free vibration analysis of FGM plates using higher order shear deformation theory," Applied Mathematical Modelling, vol. 34, no. 12, pp. 3991-4011, 2010.

[22] L. V. Tran, A. J. M. Ferreira, and H. Nguyen-Xuan, "Isogeometric analysis of functionally graded plates using higherorder shear deformation theory," Composites Part B: Engineering, vol. 51, pp. 368-383, 2013.

[23] H. Nguyen-Xuan, C. H. Thai, and T. Nguyen-Thoi, "Isogeometric finite element analysis of composite sandwich plates using a higher order shear deformation theory," Composites Part B: Engineering, vol. 55, pp. 558-574, 2013.

[24] C. H. Thai, S. Kulasegaram, L. V. Tran, and H. Nguyen-Xuan, "Generalized shear deformation theory for functionally graded isotropic and sandwich plates based on isogeometric approach," Computers \& Structures, vol. 141, pp. 94-112, 2014.

[25] A. M. Zenkour, "Benchmark trigonometric and 3-D elasticity solutions for an exponentially graded thick rectangular plate," Archive of Applied Mechanics, vol. 77, no. 4, pp. 197-214, 2007.

[26] A. M. Zenkour, "Generalized shear deformation theory for bending analysis of functionally graded plates," Applied Mathematical Modelling, vol. 30, no. 1, pp. 67-84, 2006.

[27] T. Q. Bui, T. V. Do, L. H. T. Ton et al., "On the high temperature mechanical behaviors analysis of heated functionally graded plates using FEM and a new third-order shear deformation plate theory," Composites Part B: Engineering, vol. 92, pp. 218-241, 2016. 
[28] T.-V. Do, D.-K. Nguyen, N.-D. Duc, D.-H. Doan, and T.-Q. Bui, "Analysis of bi-directional functionally graded plates by FEM and a new third-order shear deformation plate theory," Thin-Walled Structures, vol. 119, pp. 687-699, 2017.

[29] J. L. Mantari and C. Guedes Soares, "Bending analysis of thick exponentially graded plates using a new trigonometric higher order shear deformation theory," Composite Structures, vol. 94, no. 6, pp. 1991-2000, 2012.

[30] J. L. Mantari and C. Guedes Soares, "Analysis of isotropic and multilayered plates and shells by using a generalized higherorder shear deformation theory," Composite Structures, vol. 94, no. 8, pp. 2640-2656, 2012.

[31] J. L. Mantari and C. Guedes Soares, "Finite element formulation of a generalized higher order shear deformation theory for advanced composite plates," Composite Structures, vol. 96, pp. 545-553, 2013.

[32] J. L. Mantari and C. Guedes Soares, "Optimized sinusoidal higher order shear deformation theory for the analysis of functionally graded plates and shells," Composites Part B: Engineering, vol. 56, pp. 126-136, 2014.

[33] M. Arefi, E. Mohammad-Rezaei Bidgoli, R. Dimitri, M. Bacciocchi, and F. Tornabene, "Application of sinusoidal shear deformation theory and physical neutral surface to analysis of functionally graded piezoelectric plate," Composites Part B: Engineering, vol. 151, pp. 35-50, 2018.

[34] F. Tornabene, N. Francesco, and E. Viola, "Inter-laminar stress recovery procedure for doubly-curved, singly-curved, revolution shells with variable radii of curvature and plates using generalized higher-order theories and the local GDQ method," Mechanics of Advanced Materials and Structures, vol. 23, no. 9, pp. 1019-1045, 2016.

[35] I. Mechab, B. Mechab, and S. Benaissa, "Static and dynamic analysis of functionally graded plates using four-variable refined plate theory by the new function," Composites Part B: Engineering, vol. 45, no. 1, pp. 748-757, 2013.

[36] A. Benachour, H. D. Tahar, H. A. Atmane, A. Tounsi, and M. S. Ahmed, "A four variable refined plate theory for free vibrations of functionally graded plates with arbitrary gradient," Composites Part B: Engineering, vol. 42, no. 6, pp. 1386-1394, 2011.

[37] R. P. Shimpi, "Refined plate theory and its variants," $A I A A$ Journal, vol. 40, no. 1, pp. 137-146, 2002.

[38] R.-P. Shimpi and H.-G. Patel, "A two variable refined plate theory for orthotropic plate analysis," International Journal of Solids Structure, vol. 43, no. 22-23, pp. 6783-6799, 2006.

[39] H.-T. Thai and S.-E. Kim, "Analytical solution of a two variable refined plate theory for bending analysis of orthotropic Levy-type plates," International Journal of Mechanical Sciences, vol. 54, no. 1, pp. 269-276, 2012.

[40] I. Mechab, H. A. Atmane, A. Tounsi, H. A. Belhadj, and E. A. A. Bedia, "A two variable refined plate theory for the bending analysis of functionally graded plates," Acta Mechanica Sinica, vol. 26, no. 6, pp. 941-949, 2010.

[41] R. P. Shimpi, R. A. Shetty, and A. Guha, "A single variable refined theory for free vibrations of a plate using inertia related terms in displacements," European Journal of Mechanics-A/Solids, vol. 65, pp. 136-148, 2017.

[42] M. Filippi, E. Carrera, and S. Valvano, "Analysis of multilayered structures embedding viscoelastic layers by higherorder, and zig-zag plate elements," Composites Part B: Engineering, vol. 154, pp. 77-89, 2018.

[43] E. Carrera, S. Valvano, and M. Filippi, "Classical, higherorder, zig-zag and variable kinematic shell elements for the analysis of composite multilayered structures," European Journal of Mechanics-A/Solids, vol. 72, pp. 97-110, 2018.

[44] A. Alaimo, C. Orlando, and S. Valvano, "Analytical frequency response solution for composite plates embedding viscoelastic layers," Aerospace Science and Technology, vol. 92, pp. 429445, 2019. 